\title{
Aggressive Angiomyxoma of the perineum in a 50-year-old woman
}

\author{
1) Haemmerle B., 1) Keller N., 2) Monod C., 1) Schmid S. \\ 1) Gynaecology and Obstetrics, Grabs. 2) Gynaecology and Obstetrics, University Hospital, Basel
}

\section{Introduction:}

Aggressive angiomyxoma (AA) is a very rare soft tissue mesenchymal tumor (only about 250 cases described in literature), locally infiltrative with a tendency to repeated local recurrence. The first description was in $1983^{1}$. It normally occurs in the vulvovaginal region, perineum and pelvis of females in reproductive age. But there are rare cases of AA in the inguinoscrotal region of males and these usually appear in older age ${ }^{2}$. Metastases have been described in literature in three cases. These distant metastases were all to be found in the mediastinum or lungs $3,4,5$. This suggests that AA is not only a local disease. Wide local excision of the tumor is the primary management. The local recurrence rate is 25 to $47 \%$ after surgical excision $n^{6,7}$. The expression of estrogen and progesterone receptors in AA and the growth of the tumor during pregnancy suggest a hormone dependency of the tumor 6 . Descriptions of drug treatment exist with gonadotropin-releasing hormone $(\mathrm{GnRH})$ agonists in premenopausal women ${ }^{8,9}, 10,11$. As diagnostics ultrasound and computerized tomography are nonspecific. In magnetic resonance imaging the AA is distinctive. On T2-weighted scans the tumors are hyperintense, except for their lower signal internal structure which looks swirled or layered ${ }^{11,12}$. Microscopically AA is composed of many thick-walled vessels of varying size in a loose collagenous and myxoid stroma with spindle and stellate like cells9. Except for estrogen and progesterone receptors, there are also other immunohistochemical markers, such as vimentin, desmin, SMA (smooth muscle actin), CD 3413,14. Genetic findings showed a chromosomal abnormity involving chromosome 12, associated with rearrangement of $\mathrm{HMGIC}$, the architectural transcription factor, but the pathogenesis of AA is poorly understood $2,15,16,17,18$.

\section{Case report:}

A 50-year-old woman reported first in 2013 with a painless swelling of $3 \times 2 \mathrm{~cm}$ at the perineum. She underwent surgical excision. Pathologic findings reported the tumor as an AA. The margins were not tumor free. The patient didn't appear to the follow-up examinations. In 2016 she reported again with a painless swelling of $2 \times 2 \mathrm{~cm}$ at the perineum and underwent a wide local excision. The tumor infiltrated the perineal tissue deeply and for the complete resection it was necessary to resect a part of the bulbospongiosus muscle and the soft tissue of the perineum above the rectum. Then the perineal body had to be reconstructed. The wound healing was without difficulties, resection margins were tightly tumor free $(<1 \mathrm{~mm})$ and the immunohistochemical examination showed a hormonal receptor positivity of estrogen and progesterone and expression of CD31 and desmin. There was no expression of CD 34. At followups after 3 and 6 months there was no recurrence. The patient has refused a hormonal treatment so far.

\section{Conclusion:}

AA is a rare disease, but when treating women with a painless swelling in the vulvovaginal region, perineum or pelvis AA should be considered as a differential diagnosis. There is no standardised surgical or medicamentous procedure described, but complete resection seems to be pivotal. Even tumor free margins don't prevent a recurrence ${ }^{13,} 19,20$. In literature hormonal treatment in premenopausal women with GnRH agonists is discussed to reduce the extent of surgical radicality and enhance the chance of tumor free margins or as a therapy for the first or further recurrences. Descriptions of only drug treatment with gonadotropin-releasing hormone agonists without surgery and with complete radiologic remission exist ${ }^{10}$. Because the estrogen and progesterone receptors are not always strongly positive in $\mathrm{AA}$, the response to $\mathrm{GnRH}$ agonists is variable ${ }^{9,10,11}$. Aromatase inhibitors may be used in estrogen positive AA in postmenopausal women ${ }^{21}$. Hormone replacement therapy should be stopped ${ }^{11}$. Histologically there is low mitotic activity, therefore radiation therapy or chemotherapy is not really successful| ${ }^{13}{ }^{16}$. Very important because of repeated local recurrence is a long-term follow-up 6 .

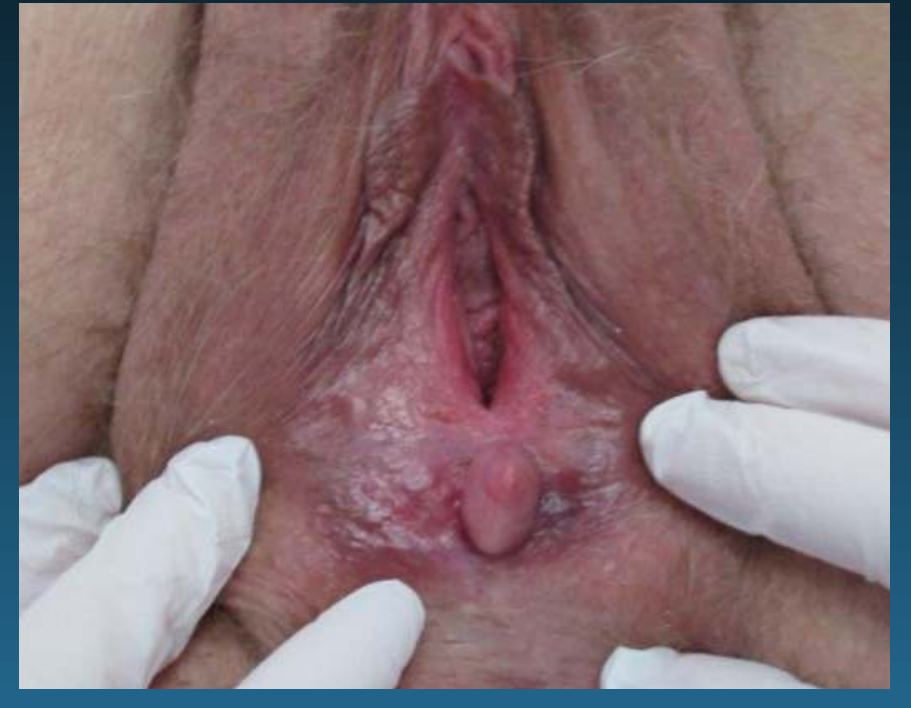

Fig. 1: Situation 3 years after the first operation

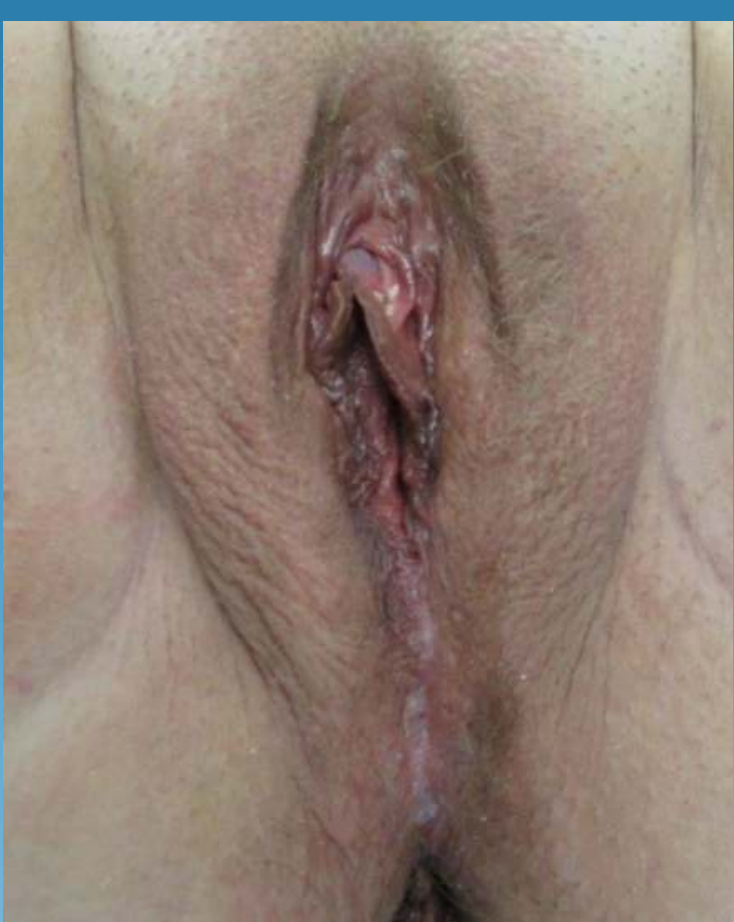

Fig. 2: Wound healing after 4 months

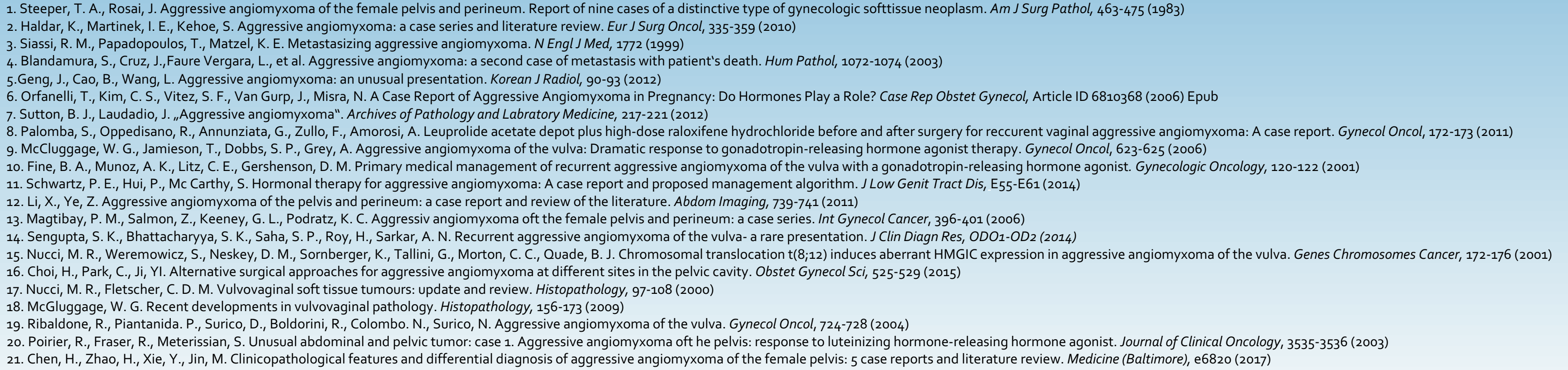

